يتتاول هذا البحث مسألة تقدير معلمات نموذج الفاريوكر ام و الذي يدعى بنمــوذج الانحلال التربيعي بواسطة أسلوب بيز الذي يتضمن المعلومات الأولية عن هذه المعلمات First Moment التي تتمل العزم الأول و العزم الثاني بشكل مصــفوفة العـزم الأول

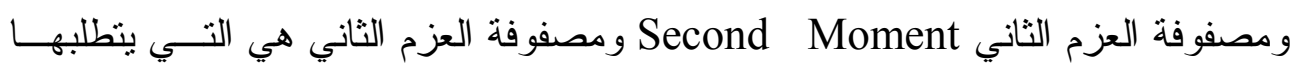
أسلوب بيز المقترح هنا ومقدر بيز المقترح في هذا البحث يسمى مقدر بيز التربيعي غير

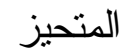

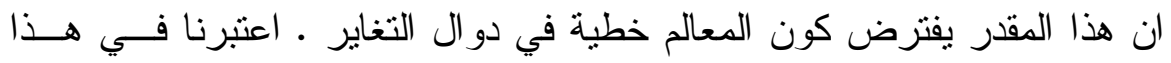
البحث دالة تغاير لمتغير مكاني يحتوي على ثلاثة معالم وبواسطة أسلوب بيز حصلنا على دونى صيغ ومعادلات كونت نظام معادلات خطية ومن خلال حل هذا النظام نحصل على تقدير بيز للمعالم الثلاث وتمت مقارنة النتائج مع نتائج تقدير بو اســــة مقــدر أصـــر معيــار

$$
\text { تربيعي غير متحيز وكانت مشجعة . }
$$

كما تمت برمجة نموذج مقدر بيز المقتر ح في هذا البحث بلغة ماثلاب وتم تطبيقه

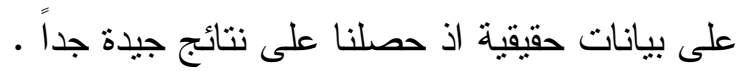

\title{
Bayesian Estimation of Parameter of Spatial Quadratic Decay Model
}

\section{ABSTRACT}

This paper deals with the problem of estimating parameters of spatial quadratic model by Bayesian technique. This technique involves the prior information of the first and second moment of the

$$
\text { " مدرس مساعد/ قسم الرياضيات - كلية التربية/جامعة الموصل : }
$$


parameters. This estimation model is called the Bayesian quadratic unbiased estimator, which is linear in the parameters. The results of estimation are compared with the estimates of minimum norm quadratic unbiased estimators and the results are encouraging.

All algorithms of computation are written by using MatLAB programming.

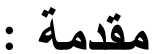

تم الحصول على بيانات و اقعية من مركز لبحوث السدود و المو ارد المائية في

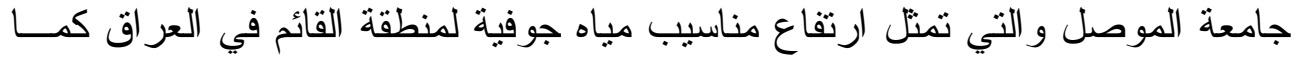
مبينة في جدول -1- تم تحليل وتوفيق نموذج التغاير الملائم لهذه البيانات $\sigma_{\mathrm{ij}}=\sigma^{2} \exp -\left(\mathrm{h}_{\mathrm{ij}} / \mathrm{a}\right)^{\mathrm{t}} \quad 0 \leq \mathrm{t} \leq 2$

بعد در استها بو اسطة دالة شبه الفاريوكر ام التقديرية

$\gamma(\mathrm{h})=\frac{1}{2 \mathrm{n}(\mathrm{h})} \sum_{\mathrm{i}=1}^{\mathrm{n}}\left(\mathrm{Z}\left(\mathrm{x}_{\mathrm{i}}\right)-\mathrm{Z}\left(\mathrm{x}_{\mathrm{i}}+\mathrm{h}\right)\right)^{2}$

حيث البيانات غير منتظمة حولت إلى شبكة منتظمة للبيانات بثــكل جـدول

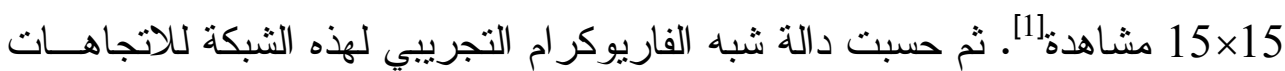
الأربعة، وبعد ذللك تم رسم معدل شبه الفاريوكر ام التجريبي و الرسم موضح في في الثكل

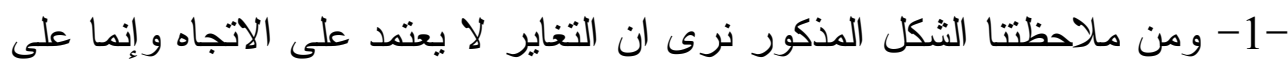

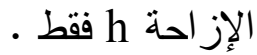

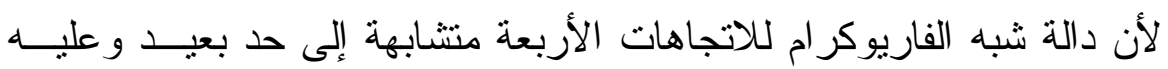

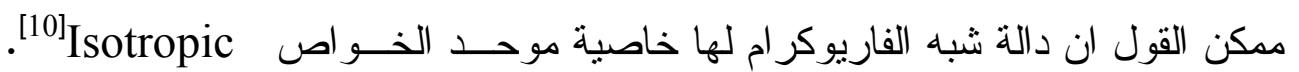

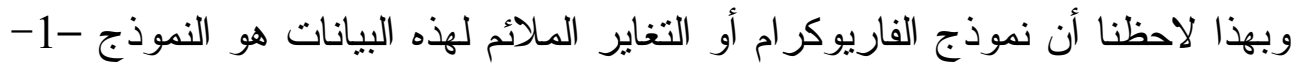

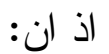

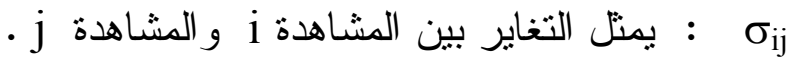
: مئل : $\sigma^{2}$ م تمثل مدى امتداد ظاهرة المياه الجوفية في المنطقة الددروسة . 
نلاحظ أن النموذج -1- نموذج تغاير غير خطي إلا ان الرسم البيــاني -1-

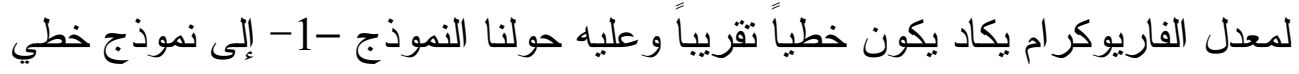
تقريبي عندما t=2 فان التقريب الخطي للنموذج -1 - سيكون :

$$
\left.\begin{array}{ll}
\sigma_{i j}=\sigma_{1}^{2}-\sigma_{2}^{2} h_{i j}^{2} & i \neq j \\
=\sigma_{1}^{2}+\sigma_{3}^{2} & i=j
\end{array}\right\}
$$

$\sigma_{1}^{2}$

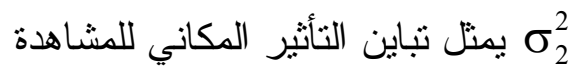

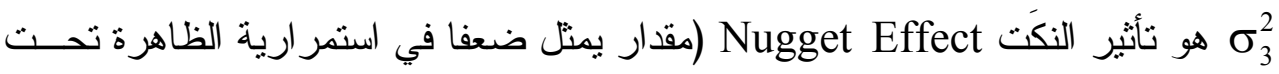

$$
\text { الدر اسة) [4 تصن. }
$$

تمثل معالم التباين الني لابد من نقدير ها للحصول على نموذج التغـاير $\sigma_{1}^{2}, \quad \sigma_{2}^{2}, \sigma_{3}^{2}$

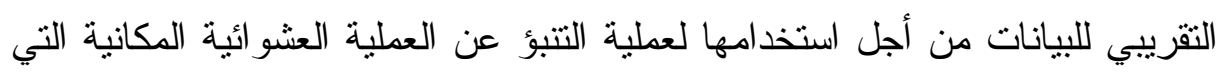

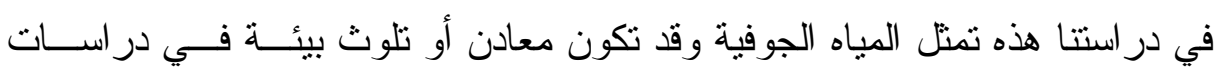

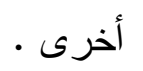
الجدول (1) :بيانات ارتفاع مناسيب المياه الجوفية لمنطقة القائم في العراق

\begin{tabular}{|l|l|l|}
\hline $\mathbf{u}(\mathbf{x})$ & $\mathbf{v}(\mathbf{x})$ & $\mathbf{Z}(\mathbf{x})$ \\
\hline 25 & 125 & 220.04 \\
\hline 125 & 125 & 220.54 \\
\hline 220 & 125 & 219.56 \\
\hline 325 & 125 & 221.26 \\
\hline 25 & 75 & 220.28 \\
\hline 125 & 75 & 219.81 \\
\hline 225 & 75 & 219.3 \\
\hline 325 & 75 & 219.92 \\
\hline 25 & 25 & 220.45 \\
\hline 125 & 25 & 220.96 \\
\hline 240 & 25 & 220.87 \\
\hline 325 & 25 & 223.04 \\
\hline 0 & 150 & 220. \\
\hline 350 & 0 & 223.3 \\
\hline
\end{tabular}




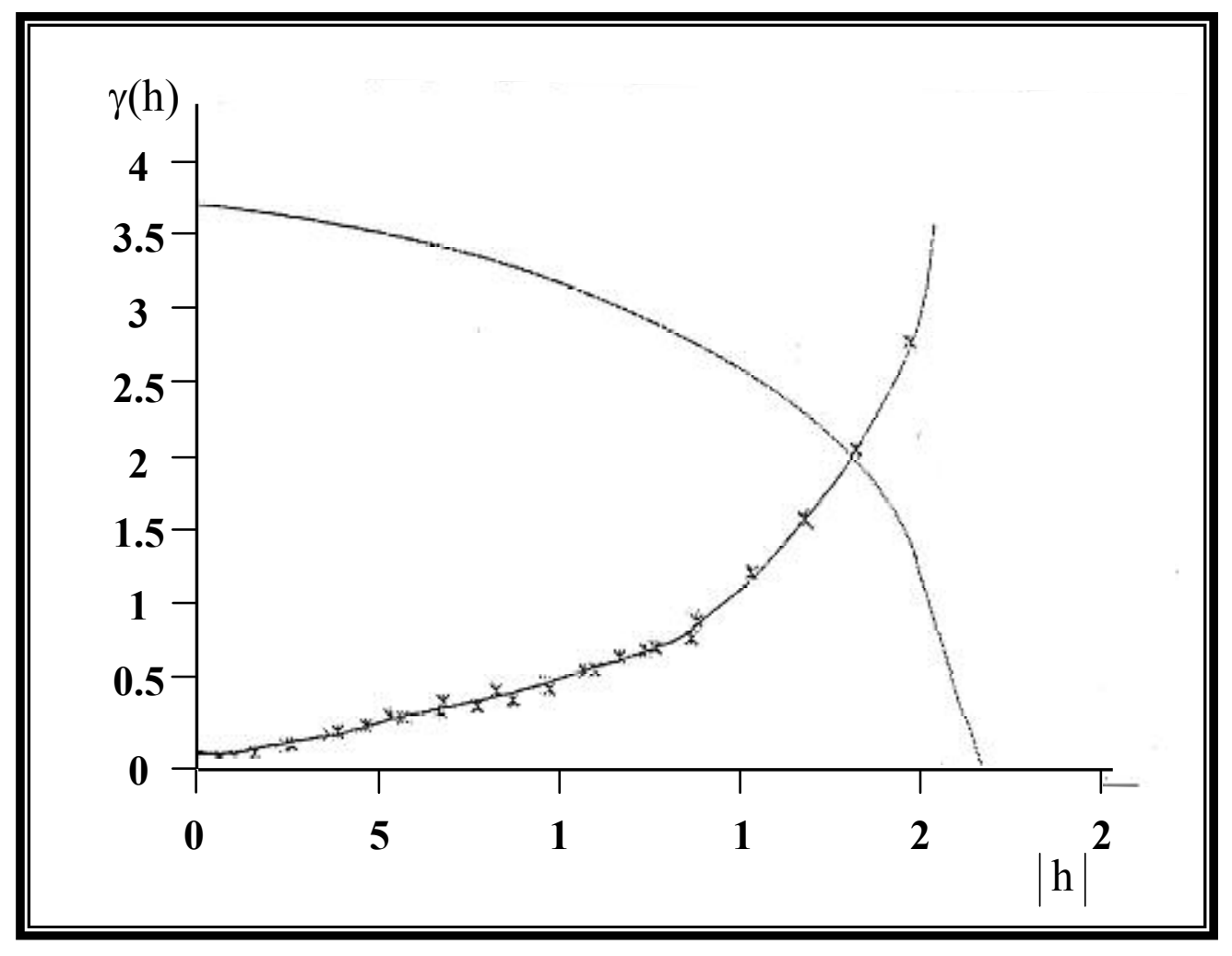

\section{الثكل (1): منحني معدل دالة شبه الفاريوكرام}

نلاحظ في النموذج -3- عندما

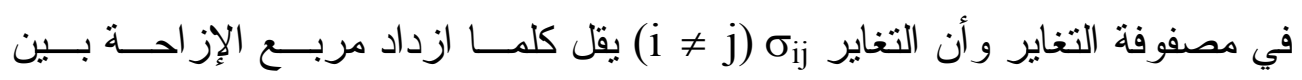

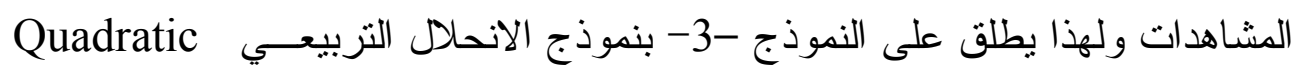
- decay Model (QD) تم تقدير المعالم

متحيز Minimum Norm Quadratic Unbiased Estimation (MINQUE) نم في هذا البحث تقدير المعلمات بو اسطة أسلوب بيز الذي سـئئي شـرحه بالتقصيل لاحقاً . 


\section{صياغة النموذج الخطي العام في الإحصاء الفراغي :}

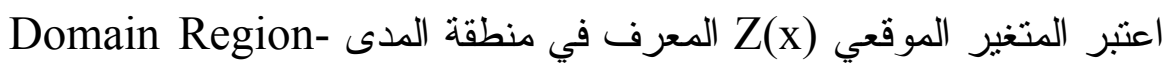

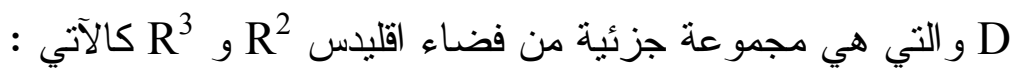
$\mathrm{Z}(\mathrm{x})=\beta^{\prime} \mathrm{f}(\mathrm{x})+\mathrm{e}(\mathrm{x}) ; \forall \mathrm{x} \in \mathrm{D}$

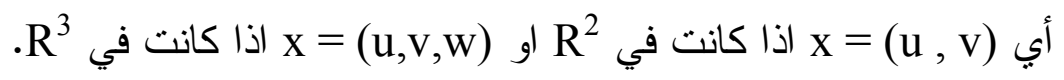

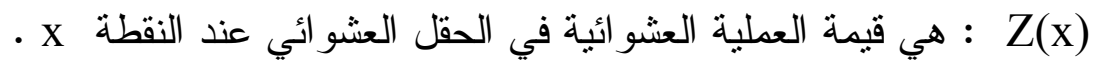

: $F(x)=\left[f_{1}(x), f_{2}(x), \ldots, f_{s}(x)\right]$

$$
\text { 及 : هي متجه بسعة sن من المعالم المجهولة }
$$

متجه عشو ائي بتوقع صفر وتباين محدود : e(x)

نفرض ان المتغير Z(x) يحقق الفرضيات الاتية :

1. $E(Z(x))=\beta^{\prime} f(x)$

2. $\mathrm{E}(\mathrm{Z}(\mathrm{x}+\mathrm{h})-\mathrm{Z}(\mathrm{x}))^{2}=2 \gamma(\mathrm{h}) ; \forall \mathrm{x}, \mathrm{x}+\mathrm{h} \in \mathrm{D}$

$$
\text { ويكون } 2 \gamma \text { موحد الخو اص Isotropic }
$$

3. $\operatorname{cov}(\mathrm{Z}(\mathrm{x}), \mathrm{Z}(\mathrm{x}+\mathrm{h}))=\mathrm{c}(\mathrm{h}) \quad \forall \mathrm{x}, \mathrm{x}+\mathrm{h} \in \mathrm{D}$

$$
\begin{aligned}
& \text { افرض أن لدينا n من المشاهدات للمتغير المكاني هي : } \\
& \text { x } 1, x_{2}, \ldots, x_{n} \text { عند المو اقل } \\
& \text { عندئذ ممكن كتابة النموذج } 4 \text { كالآتي : }
\end{aligned}
$$$$
\mathrm{Z}=\mathrm{F} \beta+\mathrm{e}
$$

اذ ان Z Z nXs مصفوفة معلومة بسعة F = (f( $\left.\left.\mathrm{x}_{1}\right), f\left(\mathrm{x}_{2}\right), \ldots, f\left(\mathrm{x}_{\mathrm{n}}\right)\right)^{\prime}$

S من المعالم المجهولة $\beta=\left(\beta_{1}, \beta_{2}, \ldots \beta_{\mathrm{s}}\right)^{\prime}$

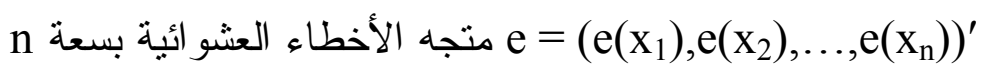


$\mathrm{C}(\mathrm{h})=\mathrm{C}(\mathrm{h} ; \theta) ; \forall \mathrm{h} \in \mathrm{D}$

$$
\text { اعتبر نموذج التغاير المعلمي الآتي : }
$$

تمثل دالة التغاير وان دالة شبه الفاريوكر ام (h) تكون :

$\gamma(\mathrm{h})=\gamma(\mathrm{h} ; \theta) ; \forall \mathrm{h} \in \mathrm{D}$

حيث ان $\theta$ متجه من المعالم المجهولة والمطلوب في هذا البحث تقدير هـــــ المعالم من البيانات بطريقة تقدير بيز وفي ضوء دالة التغاير 9 نكون مصفوفة التغاير

$\mathrm{E}\left(\mathrm{ee}^{\prime}\right)=\sum(\theta)$

\section{مقدر بيز التربيعي غير المتحيز}

\section{Bayesie Quadratic unbiased estimation}

نعتبر النموذج المعلمي للتغاير بالثكل الخطي الآتي :

$\mathrm{C}(\mathrm{h} ; \theta)=\theta_{1} \mathrm{u}_{1}(\mathrm{~h})+\theta_{2} \mathrm{u}_{2}(\mathrm{~h})+\ldots+\theta_{\mathrm{r}} \mathrm{u}_{\mathrm{r}}(\mathrm{h})$

$\forall \mathrm{i}=1,2, \ldots, r \mathrm{U}_{\mathrm{i}}(0)=1$ دو ال الارتباط و ان

列 $\theta=\left(\theta_{1}, \theta_{2}, \ldots, \theta_{r}\right)^{\prime}$ هar(Z(x))= $\theta_{1}+\theta_{2}+\ldots+\theta_{r}$ للمتغير Z تحت تأثنير النموذج 8 تكتب كالآتي : $\mathrm{U}=\theta_{1} \mathrm{U}_{1}+\theta_{2} \mathrm{U}_{2}+\ldots+\theta_{\mathrm{r}} \mathrm{U}_{\mathrm{r}}=\sum(\theta)$

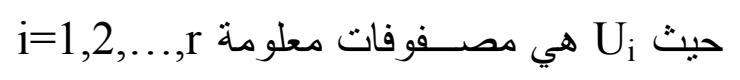

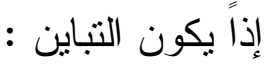

$$
\begin{aligned}
\operatorname{var}(\mathrm{Z}) & =\theta_{1} \mathrm{U}_{1}+\theta_{2} \mathrm{U}_{2}+\ldots+\theta_{\mathrm{r}} \mathrm{U}_{\mathrm{r}} \\
& =\operatorname{var}(\mathrm{e})=\sum(\theta)
\end{aligned}
$$

حيث

$\mathrm{Z}=\mathrm{F} \beta+\mathrm{e}$ نعتبر النموذج الخطي الآتي :

$\mathrm{E}(\mathrm{z})=\mathrm{F} \beta, \operatorname{var}(\mathrm{z})=\sum_{\mathrm{l}=1}^{\mathrm{r}} \theta_{\mathrm{i}} \mathrm{U}_{\mathrm{i}}=\sum(\theta)$ 


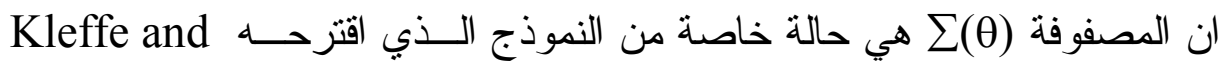
Pincus

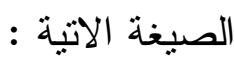

$$
\alpha(\mathrm{z})=\mathrm{b}_{1} \theta_{1}+\mathrm{b}_{2} \theta_{2}+\ldots+\mathrm{b}_{\mathrm{r}} \theta_{\mathrm{r}}=\mathrm{b}^{\prime} \theta
$$

$$
\text { بو اسطة الثنكل الثنائي ماني }
$$

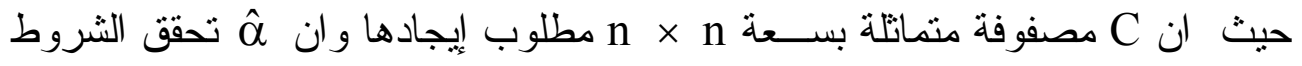
الآتية: - n

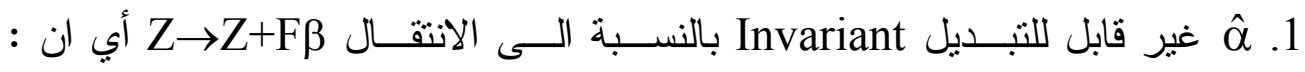

$$
. \hat{\alpha}(Z)=\hat{\alpha}(Z+F \beta)
$$

unbiased غير متحيز 2

3. تصغير دالة مخاطرة بيز

و الآن نفترض وجود دالة توزيع أولي (Apriori Distribution Function) للمعلمة و وهي P( و و و وللك فان دالة الخسارة تأخذ الصيغة الاتية :

$$
\mathrm{L}(\alpha, \hat{\alpha})=(\hat{\alpha}-\alpha)^{2}
$$

$$
\mathrm{g}(\alpha, \hat{\alpha})=\mathrm{E}(\mathrm{L}(\alpha, \hat{\alpha}))=\mathrm{E}\left((\hat{\alpha}-\alpha)^{2}\right)
$$

في حين أن دالة مخاطرة بيز B(人) تكون على الثكل التالي :

$$
\begin{aligned}
\mathrm{B}(\hat{\alpha}) & =\mathrm{E}_{\theta}(\mathrm{g}(\alpha, \hat{\alpha}))=\mathrm{E}_{\theta}\left(\mathrm{E}(\hat{\alpha}-\alpha)^{2}\right) \\
& =\int_{\theta \in \Omega} \mathrm{g}(\alpha, \hat{\alpha}) \mathrm{dP}(\theta)=\int_{\theta \in \Omega} \mathrm{E}(\hat{\alpha}-\alpha)^{2} \mathrm{dP}(\theta)
\end{aligned}
$$

بينما مقدر بيز مئم هو مقدر للمعلمة $\theta$ و الذي يجعل المخاطرة المتوقعـة أو

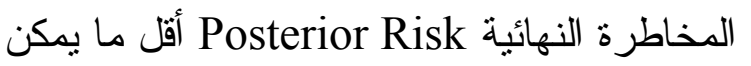

$$
\begin{aligned}
& \mathrm{B}(\hat{\alpha})=\int_{-\infty}^{\infty} \int_{-\infty}^{\infty} \ldots \ldots . \int_{-\infty}^{\infty} \mathrm{P}\left(\mathrm{Z}_{1}, \mathrm{Z}_{2}, \ldots, \mathrm{Z}_{\mathrm{n}}\right)\left[\int_{\theta \in \Omega} \mathrm{L}\left(\alpha_{1} \hat{\alpha}\right) \mathrm{P}\left(\theta / \mathrm{Z}_{1}, \mathrm{Z}_{2}, \ldots, \mathrm{Z}_{\mathrm{n}}\right) \mathrm{d} \theta\right] \mathrm{d} \mathrm{Z}_{1}, \ldots, \mathrm{dZ} \mathrm{Z}_{\mathrm{n}}
\end{aligned}
$$

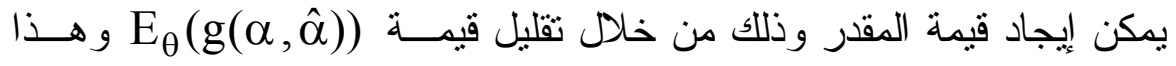

$$
\begin{aligned}
& \text { يكفي بتقليل قيمة مخاطرة بيز النهائية } \\
& \mathrm{Q}\left(\hat{\alpha}, \mathrm{Z}_{1}, \mathrm{Z}_{2}, \ldots, \mathrm{Z}_{\mathrm{n}}\right)=\int_{\theta \in \Omega} \mathrm{L}(\alpha, \hat{\alpha}) \mathrm{P}\left(\theta / \mathrm{Z}_{1}, \mathrm{Z}_{2}, \ldots, \mathrm{Z}_{\mathrm{n}}\right) \mathrm{d} \theta
\end{aligned}
$$


$\frac{\partial \mathrm{Q}\left(\hat{\alpha}, \mathrm{Z}_{1}, \mathrm{Z}_{2}, \ldots, \mathrm{Z}_{\mathrm{n}}\right)}{\partial \hat{\alpha}}=0$

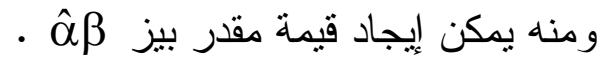

عند نوفر دالة التوزيع الأولي عن المعالم

$\mathrm{E}\left(\theta_{\mathrm{i}} \theta_{\mathrm{j}}\right)=\int_{\theta \in \Omega} \theta_{\mathrm{i}} \theta_{\mathrm{j}} \mathrm{dP}(\theta)=\mathrm{C}_{\mathrm{ij}} ; \forall_{\mathrm{i}, \mathrm{j}}=1,2, \ldots, \mathrm{r}$

اذ ان علامة التكامل في العلاقتين 20 و 18 تمثل تكامل متعدد بعدد المعالم الموجودة $\theta=\left(\theta_{1}, \theta_{2}, \ldots, \theta_{\mathrm{r}}\right)^{\prime}{ }^{\prime}$

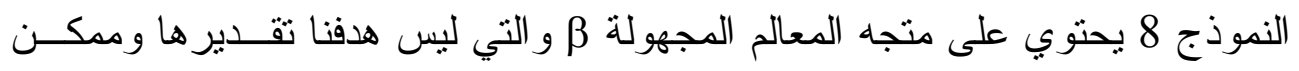

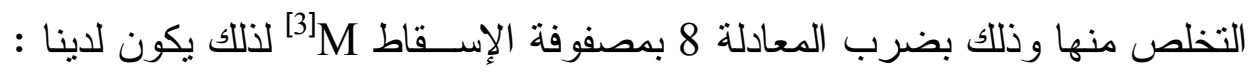
$\mathrm{MZ}=\mathrm{MF} \beta+\mathrm{Me}$

$\mathrm{MZ}=\mathrm{ME}$

الان نفرض أن y= حيث يكون y(y)=0 ولغرض البرهان نلاحظ أن : $\mathrm{E}(\mathrm{y})=\mathrm{E}(\mathrm{MZ})=\mathrm{ME}(\mathrm{Z})=\mathrm{MF} \beta=0$

$\operatorname{var}(\mathrm{y})=\operatorname{var}(\mathrm{MZ})=\mathrm{M} \operatorname{var}(\mathrm{Z}) \mathrm{M}^{\prime}$

وكنلك يكون

Mيث أن M

$\operatorname{var}(\mathrm{y})=\mathrm{M} \operatorname{var}(\mathrm{Z}) \mathrm{M}=\mathrm{M} \sum(\theta) \mathrm{M}=\mathrm{M}\left(\sum_{\mathrm{i}=1}^{\mathrm{r}} \theta_{\mathrm{i}} \mathrm{U}_{\mathrm{i}}\right) \mathrm{M}$

$$
\begin{aligned}
& =\sum_{\mathrm{i}=1}^{\mathrm{r}} \theta_{\mathrm{i}} \mathrm{MU}_{\mathrm{i}} \mathrm{M} \\
& =\sum_{\mathrm{i}=1}^{\mathrm{r}} \theta_{\mathrm{i}} \mathrm{V}_{\mathrm{i}}
\end{aligned}
$$

حيث V ونتيجه لما تقدم نحصل على النموذج الاتي :

$\mathrm{Y}=\mathrm{Me}, \mathrm{E}(\mathrm{Y})=0, \operatorname{var}(\mathrm{Y})=\sum_{\mathrm{i}=1}^{\mathrm{r}} \theta_{\mathrm{i}} \mathrm{V}_{\mathrm{i}}=\mathrm{V}(\theta)$

اذ نلاحظ أن فㄹ إلى

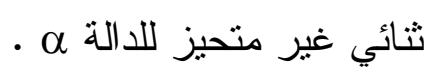


شروط التقدير للثكل الثنائي Y'A Y

1. عدم التحزي:

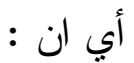

$\mathrm{E}\left(\mathrm{Y}^{\prime} \mathrm{AY}\right)=\alpha=\mathrm{b}^{\prime} \theta$

$$
\begin{aligned}
\mathrm{E}\left(\mathrm{Y}^{\prime} \mathrm{AY}\right)=\mathrm{E}\left(\operatorname{tr} \mathrm{Y}^{\prime} \mathrm{AY}\right) & =\mathrm{E}\left(\operatorname{tr} \mathrm{AYY} \mathrm{Y}^{\prime}\right) \\
& =\operatorname{trAE}\left(\mathrm{YY}^{\prime}\right) \\
& =\operatorname{trAE}\left(\mathrm{YY}^{\prime}\right) \\
& =\operatorname{trA} \operatorname{var}(\mathrm{y})=\operatorname{trA} \sum_{\mathrm{i}=1}^{\mathrm{r}} \theta_{\mathrm{i}} \mathrm{V}_{\mathrm{i}} \\
& =\sum_{\mathrm{i}=1}^{\mathrm{r}} \theta_{\mathrm{i}} \operatorname{trAV_{i}}
\end{aligned}
$$

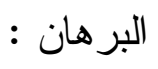

يكون غير متحيز بالنسبة إلى $\alpha$ إذا وفقط إذا

$$
\operatorname{tr} A v_{i}=b_{i} ; i=1,2, \ldots, r
$$

$$
\begin{aligned}
\mathrm{B}(\hat{\alpha}) & =\int_{\theta \in \Omega} \mathrm{E}(\hat{\alpha}-\alpha)^{2} \mathrm{dp}(\theta)=\mathrm{E} \theta\left(\mathrm{E}(\hat{\alpha}-\alpha)^{2}\right) \\
& =\mathrm{E} \theta(\operatorname{var}(\hat{\alpha}))=\mathrm{E} \theta\left(\operatorname{var}\left(\mathrm{Y}^{\prime} \mathrm{AY}\right)\right) \\
& =\mathrm{E} \theta(2 \operatorname{trAV}(\theta) \mathrm{AV}(\theta)) \\
& =\mathrm{E} \theta\left(2 \operatorname{tr} \mathrm{A} \sum_{\mathrm{i}=1}^{\mathrm{r}} \theta_{\mathrm{i}} \mathrm{V}_{\mathrm{i}} \mathrm{A} \sum_{\mathrm{j}=1}^{\mathrm{r}} \theta_{\mathrm{j}} \mathrm{V}_{\mathrm{j}}\right) \\
& =\mathrm{E} \theta\left(2 \sum_{\mathrm{i}} \sum_{\mathrm{j}} \theta_{\mathrm{i}} \theta_{\mathrm{j}} \operatorname{tr} \mathrm{AV}_{\mathrm{i}} \mathrm{AV} \mathrm{V}_{\mathrm{j}}\right) \\
& =2 \sum_{\mathrm{i}} \sum_{\mathrm{j}} \mathrm{E}(\theta)\left(\theta_{\mathrm{i}} \theta_{\mathrm{j}}\right) \operatorname{tr} \mathrm{AV} \mathrm{V}_{\mathrm{i}} \mathrm{AV} \mathrm{V}_{\mathrm{j}} \\
& =2 \sum_{\mathrm{i}} \sum_{\mathrm{j}} \mathrm{C}_{\mathrm{ij}} \operatorname{trA} \mathrm{tr}_{\mathrm{i}} \mathrm{Av}_{\mathrm{j}}
\end{aligned}
$$

المعلومات الأولية الني نحتاج اليها لتقدير متجه المعـالم '

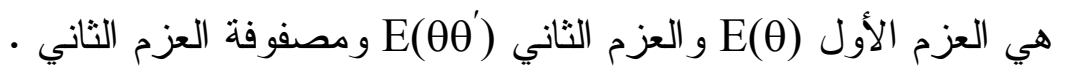

$$
\left.\mathrm{C}=\mathrm{E}\left(\theta \theta^{\prime}\right)=\operatorname{var}(\theta)+\mathrm{E}(\theta) \mathrm{E}\left(\theta^{\prime}\right)\right\}
$$




$$
\mathrm{C}=\sqrt{\mathrm{C}} \sqrt{\mathrm{C}}=\mathrm{RR}
$$

حيث ان $\mathrm{C}=\sqrt{\mathrm{C}} \mathrm{C}$ جذر المصفوفة يمكن تمثيل المصفوفة C بالثكل الاتي:

$$
\mathrm{C}=\left(\mathrm{C}_{\mathrm{ij}}\right)=\left(\sum_{\mathrm{k}=1}^{\mathrm{r}} \mathrm{r}_{\mathrm{ik}} \mathrm{r}_{\mathrm{kj}}\right) \quad \forall_{1, \mathrm{j}}=1,2, \ldots, \mathrm{r}
$$

نعوض 24 في العلاقة 22 فنحصل على العلاقة الاتية:

$$
\begin{aligned}
& \mathrm{B}(\hat{\alpha})=2 \sum_{\mathrm{i}=1}^{\mathrm{r}} \sum_{j=1}^{\mathrm{r}} \sum_{\mathrm{k}=1}^{\mathrm{r}} \mathrm{r}_{\mathrm{ik}} \mathrm{r}_{\mathrm{kj}} \operatorname{tr} \mathrm{A} \mathrm{v}_{\mathrm{i}} \mathrm{A} \mathrm{v}_{\mathrm{j}} \\
& =2 \sum_{\mathrm{i}=1}^{\mathrm{r}} \operatorname{tr} \mathrm{A}\left(\sum_{\mathrm{i}=1}^{\mathrm{r}} \mathrm{r}_{\mathrm{ik}} \mathrm{v}_{\mathrm{i}}\right) \mathrm{A}\left(\sum_{\mathrm{j}=1}^{\mathrm{r}} \mathrm{r}_{\mathrm{kj}} \mathrm{v}_{\mathrm{j}}\right) \\
& \mathrm{B}(\hat{\alpha})=2 \sum_{\mathrm{k}=1}^{\mathrm{r}} \operatorname{trAT}_{\mathrm{k}} \mathrm{AT}_{\mathrm{k}}
\end{aligned}
$$

$$
\mathrm{T}_{\mathrm{k}}=\sum_{\mathrm{i}=1}^{\mathrm{r}} \mathrm{r}_{\mathrm{ik}} \mathrm{v}_{\mathrm{i}}
$$

الآن سوف نستخدم طريقة لاكر انج لحل العلاقة 25 وفقاً إلى شــروط عـدم

التحيز 21 للحصول على قيمة قصوى صغرى ـ من أجل ذلك نفرض أن :

$\mathrm{N}=2 \sum_{\mathrm{k}=1}^{\mathrm{r}} \operatorname{trAT}_{\mathrm{k}} \mathrm{AT}_{\mathrm{k}}+4 \sum_{\mathrm{i}=1}^{\mathrm{r}} \delta\left(\operatorname{tr} \mathrm{Av}_{\mathrm{i}}-\mathrm{b}_{\mathrm{i}}\right)$

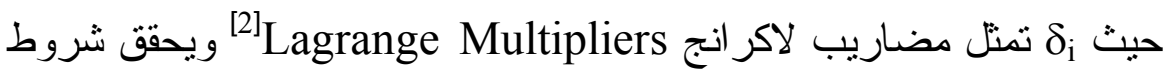

$$
\text { عدم التحيز } 21 \text {. }
$$

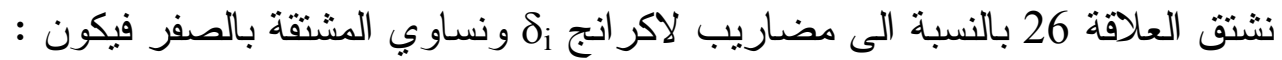
$\frac{\partial \mathrm{N}}{\partial \delta_{\mathrm{i}}}=\operatorname{tr} A \mathrm{v}_{\mathrm{i}}-\mathrm{b}_{\mathrm{i}}=0 \quad \mathrm{i}=1,2, \ldots, \mathrm{r}$

$$
\operatorname{tr} A v_{i}=b_{i} \quad i=1,2, \ldots, r
$$


A ان المعادلتين 27 و 28 بشكل مصفوفات و المجهــول فيهـــا المصــفوفة ومضاريب لاكر انج $\delta_{i}$ المجاهيل نحول هذه المعادلات من صيخ نظام المصفوفات إلى نظام معادلات خطيــة ونستخدم من أجل ذلك عملية كرونيكر Kronecker product و عملية المتجه : Operation

$$
\begin{aligned}
& \left(\sum_{\mathrm{k}=1}^{\mathrm{r}} \mathrm{T}_{\mathrm{k}} \otimes \mathrm{T}_{\mathrm{k}}\right) \operatorname{vec} \mathrm{A}+\sum_{\mathrm{i}=1}^{\mathrm{r}} \delta_{\mathrm{i}} \operatorname{vec} \mathrm{V}_{\mathrm{i}}=0 \\
& \left(\operatorname{vec} \mathrm{V}_{\mathrm{i}}\right)^{\prime} \operatorname{vec} \mathrm{A}=\mathrm{b}_{\mathrm{i}} \quad \mathrm{i}=1,2, \ldots, \mathrm{r}
\end{aligned}
$$
$\mathrm{W}=\sum_{\mathrm{k}=1}^{\mathrm{r}} \mathrm{T}_{\mathrm{k}} \otimes \mathrm{T}_{\mathrm{k}}$

إذاً تصبح العلاقة (29) بالصيغة الاتية :

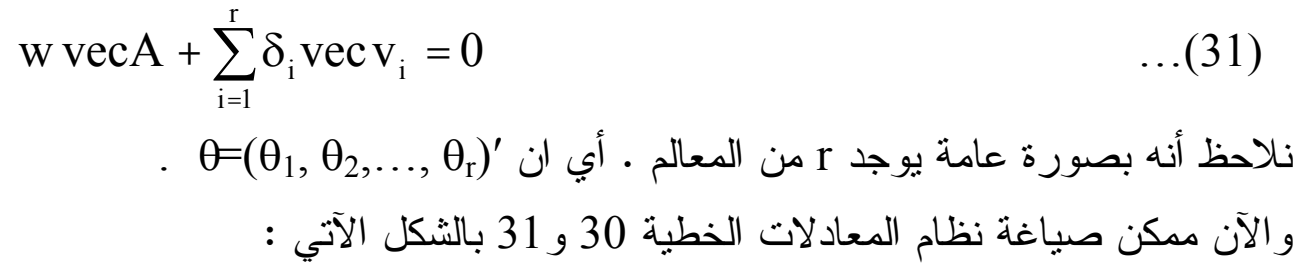

$\left(\begin{array}{cccc|c}\operatorname{vec}_{1} & \operatorname{vec}_{2} & \ldots & \operatorname{vec}_{\mathrm{r}} & \mathrm{w} \\ \hline 0 & 0 & & 0 & \operatorname{vec} \mathrm{v}_{1}^{\prime} \\ 0 & 0 & & 0 & \operatorname{vec} \mathrm{v}_{2}^{\prime} \\ 0 & 0 & \ldots & 0 & \operatorname{vec} \mathrm{v}_{\mathrm{r}}^{\prime}\end{array}\right)\left(\begin{array}{c}\delta_{1} \\ \delta_{2} \\ \vdots \\ \delta_{\mathrm{r}} \\ \hdashline \operatorname{vec} \mathrm{A}\end{array}\right)=\left(\begin{array}{c}0 \\ 0 \\ \vdots \\ 0 \\ \hdashline \mathrm{b}_{1} \\ \mathrm{~b}_{2} \\ \vdots \\ \mathrm{b}_{\mathrm{r}}\end{array}\right)$

أما في الحالة التي نتتاولها في هذا البحث فيكون عدد المعــالم فيهــا r=3 . و عندما r=3 فان متجه المعالم يكون بالصيغة الاتية ' إذاً يصبح نظام المعادلات 31 و 30 كالآتي :

$$
\begin{aligned}
& \mathrm{w} \operatorname{vec} A+\sum_{i=1}^{3} \delta_{i} \operatorname{vec} v_{i}=0 \\
& \left(\operatorname{vec}_{i}\right)^{\prime} \operatorname{vec} A=b_{i} \quad i=1,2,3
\end{aligned}
$$


لذلك يمكن صياغة نظام المعادلات الخطية 34 و 35 بالثكل الآتي : (d $\left(\begin{array}{ccc|c}\operatorname{vec} \mathrm{v}_{1} & \operatorname{vec}_{2} & \operatorname{vec} \mathrm{v}_{3} & \mathrm{w} \\ \hline 0 & 0 & 0 & \operatorname{vec} \mathrm{v}_{1}^{\prime} \\ 0 & 0 & 0 & \operatorname{vec} \mathrm{v}_{2}^{\prime} \\ 0 & 0 & 0 & \operatorname{vec} \mathrm{v}_{3}^{\prime}\end{array}\right)\left(\begin{array}{c}\delta_{1}^{\prime} \\ \delta_{2}^{\prime} \\ \delta_{3}^{\prime} \\ \hdashline \operatorname{vec} \mathrm{A}\end{array}\right)=\left(\begin{array}{c}0 \\ 0 \\ 0 \\ -\cdots \\ \mathrm{b}_{1} \\ \mathrm{~b}_{2} \\ \mathrm{~b}_{3}\end{array}\right)$

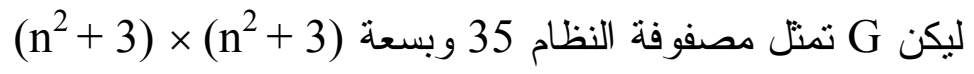

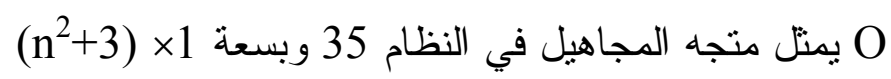

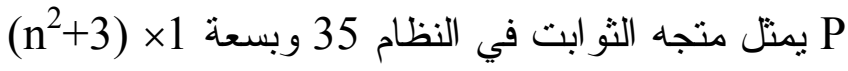

$$
\begin{aligned}
& \text { وبهذا يمكن صياغة نظام المعادلات } 35 \text { بالعلاقة الآتية : }
\end{aligned}
$$

$\mathrm{GO}=\mathrm{P}$

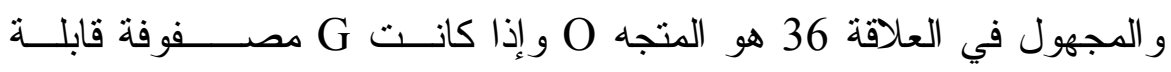

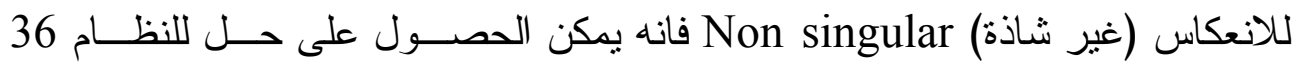
$\mathrm{O}=\mathrm{G}^{-1} \mathrm{P}$

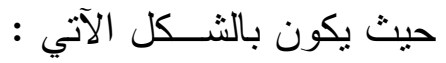

ومن خلال إيجاد قيم متجه المجاهيل O و الني تحتوي على

يمكن إيجاد المصفوفة A و التي من خلالاها يمكن الحصول على تقدير المعالم الثلاث

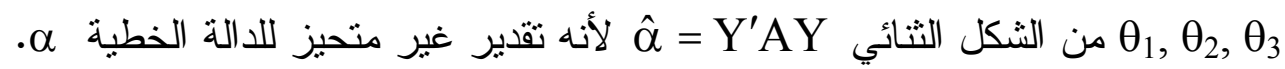

$$
\alpha=b_{1} \theta_{1}+b_{2} \theta_{2}+b_{3} \theta_{3}
$$

إذا وفقط إذا

$$
\operatorname{tr} \mathrm{Av}_{\mathrm{i}}=\mathrm{b}_{\mathrm{i}} \quad \mathrm{i}=1,2,3
$$

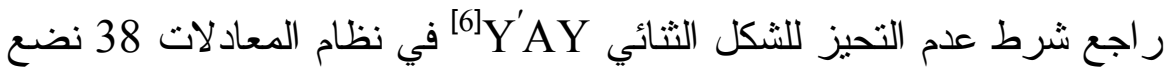

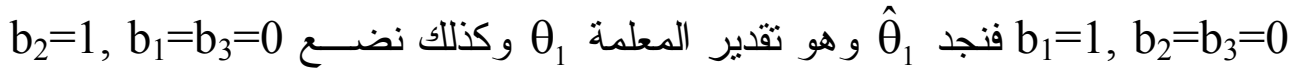

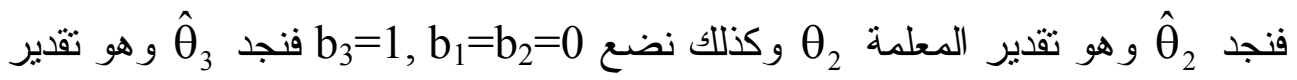
المعلمة $\theta_{3}$ ـ و إذا ما أردنا تقدير آخر مثنلاً كلاً من 


\section{الجاتب التطبيقي : تطبيق مقدر بيز التربيعي غير المتحيز}

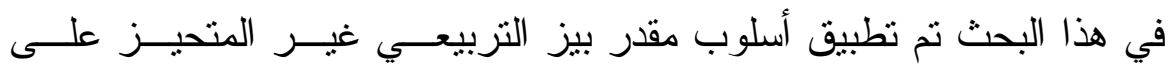

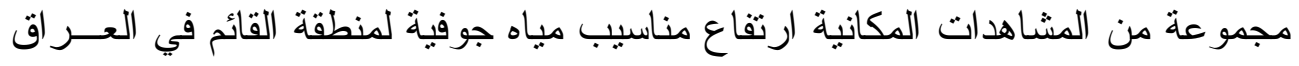

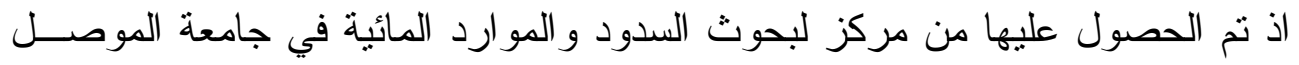

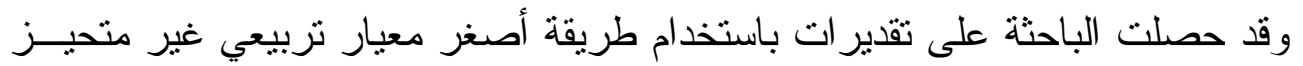

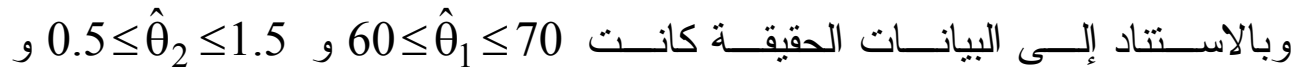
و $0.0 \leq \hat{\theta}_{3} \leq 0.1$

$$
\begin{array}{ll}
\mathrm{P}_{1}\left(\theta_{1}\right) \frac{1}{70-60}=0.1 & 60 \leq \hat{\theta}_{1} \leq 70 \\
\mathrm{P}_{2}\left(\theta_{2}\right) \frac{1}{1.5-0.5}=1 & 0.5 \leq \hat{\theta}_{2} \leq 1.5 \\
\mathrm{P}_{3}\left(\theta_{3}\right) \frac{1}{0.1-0.0}=10 & 0.0 \leq \hat{\theta}_{3} \leq 0.1
\end{array}
$$$$
\mathrm{P}_{1}\left(\theta_{1}, \theta_{2}, \theta_{3}\right)=\mathrm{P}_{1}\left(\theta_{1}\right) \mathrm{P}_{2}\left(\theta_{2}\right) \mathrm{P}_{3}\left(\theta_{3}\right)
$$$$
\text { ان توزيع }
$$$$
\operatorname{cov}\left(\theta_{1}, \theta_{2}, \theta_{3}\right)=0
$$$$
\mathrm{E}\left(\theta_{1}\right)=\frac{70+60}{2}=65
$$$$
\mathrm{E}\left(\theta_{2}\right)=\frac{1.5+0.5}{2}=1.0
$$$$
\mathrm{E}\left(\theta_{3}\right)=\frac{0.1+0.0}{2}=0.05
$$$$
\operatorname{var}\left(\theta_{1}\right)=\frac{(70-60)^{2}}{12}=8.33
$$$$
\operatorname{var}\left(\theta_{2}\right)=\frac{1}{12}=0.0833
$$ 


$$
\begin{aligned}
& \operatorname{var}\left(\theta_{3}\right)=\frac{(0.1)^{2}}{12}=0.000833 \\
& \mathrm{C}=\mathrm{E}(\theta) \mathrm{E}\left(\theta^{\prime}\right)+\operatorname{var}(\theta) \\
& =\left(\begin{array}{c}
65 \\
1 \\
0.05
\end{array}\right)\left(\begin{array}{lll}
65 & 1 & 0.05
\end{array}\right)+\left(\begin{array}{ccc}
8.33 & 0 & 0 \\
0 & 0.0833 & 0 \\
0 & 0 & 0.000833
\end{array}\right) \\
& =\left(\begin{array}{ccc}
4225 & 65 & 3.25 \\
65 & 1 & 0.05 \\
3.25 & 0.05 & 0.0025
\end{array}\right)+\left(\begin{array}{ccc}
8.33 & 0 & 0 \\
0 & 0.0833 & 0 \\
0 & 0 & 0.000833
\end{array}\right) \\
& \mathrm{C}=\left(\begin{array}{ccc}
4233.33 & 65 & 3.25 \\
65 & 1.0833 & 0.05 \\
3.25 & 0.05 & 0.003333
\end{array}\right) \\
& \text { بما أن الجذر التربيعي للمصفوفة C هنا يكون :- }
\end{aligned}
$$$$
R=\left(\begin{array}{ccc}
65.056 & 0.99443 & 0.049918 \\
0.99443 & 0.3073 & 0.00107 \\
0.049918 & 0.00107 & 0.028989
\end{array}\right)
$$

فقد حصلنا على قيمة تقدير BAQUE للمعالم الجدول -2-: نتائج تقديرات بواسطة مقدر بيز التربيعي غير المتحيز

\begin{tabular}{|l|l|l|}
\hline$\hat{\theta}_{1}$ & $\hat{\theta}_{2}$ & $\hat{\theta}_{3}$ \\
\hline $0.897 \times 10^{-2}$ & $4.27 \times 10^{-2}$ & -352.69 \\
\hline
\end{tabular}

أما النتائج التي حصلت عليها الباحثة باستخدام طريقة أصغر معيار تربيعسي

$$
\text { غير متحيز كما مبين في الجدول -3-31[ن. }
$$
الجدول -3-: نتائج تقديرات بواسطة أصغر معيار تربيعي غيرمتحيز

\begin{tabular}{|l|l|l|}
\hline$\hat{\theta}_{1}$ & $\hat{\theta}_{2}$ & $\hat{\theta}_{3}$ \\
\hline $7.036 \times 10$ & $5.827 \times 10^{-2}$ & $-7.345 \times 10^{-4}$ \\
\hline
\end{tabular}


1. من الملاحظ بأن التقدير ات قريبة من بعضها مما يدل على أنه يمكن اســتخدام

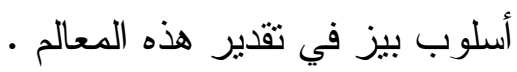

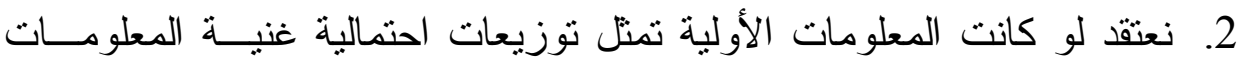

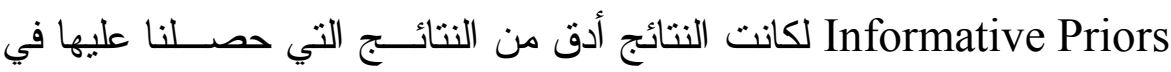

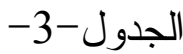

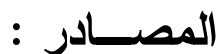

1. جابر ، عدنان شخي ، ضوية سلمان حسن (1988) ، مقدمسة فـي بحسوث العمليات ، وزارة التعليم العالي و البحث العلمي ، جامعة بخداد .

2. عبد الرحمن ، نبال صباح (2001) نموذج تغاير مكاني خطي مـــ تطبيـق رسالة ماجستير غير منشورة ، جامعة الموصل ، موصل ، العر اق . 3. يونس (1996) تقدير بيز لدو ال التغاير الفر اغي بمعلمتين وثناث معالم رسالة ماجنتير غير منشورة ، جامعة الموصل ، موصل ، العر اق •

4. Cressie, N. (1993): Statistics for Spatial Data. John Wiley, NewYork.

5. Delfiner, P. (1976): Linear Estimation of Nonstationary spatial phenomena. In: Guarascio, M., David and Huijbregts, C. (Ed.). advances Geostatistics in the Mining Industry Reidel, D. publishing Co. Holland, pp. 49.68.

6. Davies, W.S. (2002) : Quantitative Methods, Bayesian Inference. Progressin Human Geography, Vol. 26, 4, P. 553.

7. Diggle, P. J. (2002): Bayesian Inference in Gaussian Model Geostatistics.Geographical and Enviro Mental Modeling, Vol. 6, 2, P. 129.

8. Hogg, R. V. and Craig A. T. (1978): Introduction to mathematical statistics. Macmillan publishing Co., Inc. New York.

9. Kleffe, J. and Pincus, R. (1974): Bayes and Best Quadratic unbiased Estimators for Parameters of the covariance Matrix 
[196] [ ] [ تقدير بيز لمعلمات نموذج الانحلال التربيعي المكاني

in a Normal Linear Model. Math. Operations forsch. U. Statist., 5, Heft 1, S. 43-67.

10. Krige, D. G. (1976): Some Basic Consideration in the Application of Geostatistics to the valuation of ore in south African Gold Mines, Journal of the South African Institute of Mining and Metallurgy. 383-391.

11. Marshall, R. J. and Mardia, K. V. (1985): Minimum Norm Quadratic Estimation of components of spatial covariance. Math. Geol., Vol. 17, No.5, pp.517-525.

12. Rao, C. R. and Kleffe, J. (1988): Estimation of Variance Components and Application. Worth-Holland, New York. 